\title{
RUND FORMS OVER REAL ALGEBRAIC FUNCTION FIELDS IN ONE VARIABLE
}

\author{
RICHARD ELMAN ${ }^{1}$
}

ABSTRACr. The isometry types of rund quadratic forms over an arbitrary real algebraic function field in one variable are completely determined.

Using his theory of rund forms, Witt was able to simplify proofs for some of the well-known structure theorems for the Witt ring $W(F)$ (see [6]). Thus the determination of all rund forms over a field $F$ became a desirable goal. Using local-global methods, Hsia and Johnson were able to compute all rund forms over a global field (see [4]) and over $R(t)$ (see [5]). In this note, using the theory of Pfister forms, we determine all rund forms over an algebraic extension field $F$ over $R(t)$. In particular, we show that an even dimensional rund form over $F$ is isometric to a quadratic form of the type $r\langle 1, x\rangle \perp\langle 1, w x\rangle$ for some integer $r \geqq 0$, where $x \in \dot{F}$ and $w$ is a sum of two squares in $\dot{F}$.

The notation in this note will follow [3]. Thus by a field $F$, we shall mean one whose characteristic is different from two. A quadratic form $\otimes_{i=1}^{n}\left\langle 1, x_{i}\right\rangle, x_{i} \in \dot{F}=F-\{0\}$ will be called an $n$-fold Pfister form and be notated by $\left\langle\left\langle x_{1}, \cdots, x_{n}\right\rangle\right\rangle$. The $n$-fold Pfister forms generate $I^{n} F$ as an abelian group, where $I F$ is the ideal in the Witt ring $W(F)$ consisting of (nonsingular) even dimensional quadratic forms. If $q$ is a quadratic form, we shall write $D_{F}(q)=D(q)$ for the set of nonzero values represented by $q$, and $G_{F}(q)=G(q)$ for the group of similarity factors $\{x \in \dot{F}:\langle x\rangle q \cong q\}$ of $q$. A nonsingular quadratic form $q$ is called rund over $F$ if either $D(q)=$ $G(q)$ and $q$ is anisotropic or $q$ is hyperbolic. Thus if $q$ is rund, $\langle\langle-x\rangle\rangle q=0$ (in $W(F)$ ) for all $x \in D(q)$. It is well known that Pfister forms are rund, and we shall use this fact implicitly throughout this note.

We begin by classifying all odd dimensional rund forms over an arbitrary field.

LEMMA. Let $F$ be an arbitrary field. If an odd dimensional form $q$ over $F$ is rund then $q \cong(2 r+1)\langle 1\rangle$ for some integer $r \geqq 0$. The form $\langle 1\rangle$ is always

Received by the editors February 5, 1973.

AMS (MOS) subject classifications (1970). Primary 15A63.

Key words and phrases. Quadratic forms, rund forms, Pfister forms, Witt ring.

${ }^{1}$ Partially supported by NSF GP 37508 X.

(c) American Mathematical Society 1973 
rund over $F$. A form $(2 r+1)\langle 1\rangle, r$ a positive integer, is rund over $F$ iff $(2 n+1)\langle 1\rangle$ is rund for any integer $n \geqq 0$ iff $F$ is formally real and pythagorean.

Proof. If $q$ is rund over $F$ then $D(q)=G(q)$. Thus, if $\operatorname{dim} q$ is odd, we must have $\langle\operatorname{det} q\rangle \cong\langle a \cdot \operatorname{det} q\rangle$ for all $a \in D(q)$. Consequently, $D(q)=\dot{F}^{2}$. The first two statements now follow. If for some $r \geqq 1,(2 r+1)\langle 1\rangle$ is rund, then $D((2 r+1)\langle 1\rangle)=\dot{F}^{2}$. Hence $F$ is pythagorean. If $F$ is not formally real then every form of dimension $>1$ must be isotropic. But there can exist no hyperbolic odd dimensional forms over $F$, hence $F$ must be formally real. Conversely, if $F$ is formally real and pythagorean then $n\langle 1\rangle$ is clearly rund for any integer $n \geqq 1$. Q.E.D.

Since any isotropic form over $F$ is rund iff it is hyperbolic, the lemma reduces the determination of rund forms to the classification of even dimensional, anisotropic rund forms. In general, such a classification is unknown. We shall, however, determine all rund forms over $F$ if $\operatorname{tr} \operatorname{deg}_{R} F=1$. If $F$ is such a field then $F(\sqrt{ }-1)$ must be a $C_{1}$-field by the theorem of Tsen-Lang and hence must also satisfy $I^{2} F(\sqrt{ }-1)=0$. Since our results hold in this more general circumstance, we shall only assume that $I^{2} F(\sqrt{ }-1)=0$.

We begin with some preliminary results.

HAUPTSATZ [1]. If $q \in I^{n} F$ is anisotropic then $\operatorname{dim} q \geqq 2^{n}$.

THEOREM 1. Suppose that $I^{2} F(\sqrt{ }-1)=0$. Then the following statements are valid over $F$ :

(1) If $q \in I F$ is anisotropic, then $q \cong\langle x\rangle\langle\langle\operatorname{det} q\rangle\rangle \perp 2 q_{1}$ for some form $q_{1}$ over $F$ and some $x \in \dot{F}$.

(2) $I^{2} F=2 I F$ is torsion-free. In particular, if $\varphi$ is any $n$-fold Pfister form over $F$, then there exists a $z \in \dot{F}$ such that $\varphi \cong 2^{n-1}\langle\langle z\rangle\rangle$.

(3) If $\varphi$ is any $n$-fold Pfister form over $F$, then $D(\varphi)=D(r \varphi)$ for any positive integer $r$. In particular, if $q \in I F$ then $D(q)=D(r q)$ for any positive integer $r$. Moreover, any sum of squares in $F$ is a sum of two squares in $F$.

(4) If $\varphi$ and $\tau$ are two $n$-fold Pfister forms over $F$ with $n \geqq 2$, then $\varphi \cong \tau$ iff $D(\varphi)=D(\tau)$.

Proof. Let $\mu$ be any 3-dimensional form over $F(\sqrt{ }-1)$. Then $\mu \perp\langle\operatorname{det} \mu\rangle \in I^{2} F(\sqrt{ }-1)$. Hence if $I^{2} F(\sqrt{ }-1)=0$ any 3-dimensional form $\mu$ over $F(\sqrt{ }-1)$ is isometric to $\langle-\operatorname{det} \mu, 1,-1\rangle$, an isotropic form.

(1) If $\varphi$ is anisotropic over $F$ and $\operatorname{dim} \varphi>2$, then $\varphi$ becomes isotropic over $F(\sqrt{ }-1)$ by the above remark. Consequently, $q \cong\langle a, b\rangle \perp 2 q_{1}$ for some form $q_{1}$ and $a, b \in \dot{F}$ by (2.2.9) of [6]. But det $q=a b$ so (1) follows.

(2) By (4.11) of [3], any anisotropic form $q$ which is torsion in $W(F)$ must satisfy $\operatorname{dim} q \leqq 2$. Thus $I^{2} F$ is torsion-free by the Hauptsatz. Since a form $q$ lies in $I^{2} F$ iff $\operatorname{dim} q$ is even and $\operatorname{det} q=(-1)^{(\operatorname{dim} a) / 2}$, (1) implies that 
$I^{2} F=2 I F$. Moreover, (1) implies that any 2-fold Pfister form $\varphi \cong\langle x, x, y, y\rangle$ for some $x, y \in \dot{F}$. But Pfister forms are rund, so $\varphi \cong 2\langle\langle x y\rangle\rangle$. Consequently, if $\tau$ is any $n$-fold Pfister form, induction implies that there exists a $z \in \dot{F}$ such that $\tau \cong 2^{n-1}\langle\langle z\rangle\rangle$.

(3) If $\varphi$ is an $n$-fold Pfister form, we may write $\varphi \cong 2^{n-1}\langle\langle z\rangle\rangle$ for some $z \in \dot{F}$ by (2). Suppose $y \in D(2 \varphi)$. Then $2\langle\langle-y\rangle\rangle \varphi=0$ in $W(F)$ and $\langle\langle-y, z\rangle\rangle \in I^{2} F$ is torsion. By $(2),\langle\langle-y, z\rangle\rangle=0$, i.e. $y \in D(\langle\langle z\rangle\rangle)$. Hence $D(2 \varphi) \subset D(\varphi)$. (3) is now easily deduced.

(4) If $\varphi, \tau$ are two $n$-fold Pfister forms, then $\varphi \cong 2^{n-1}\langle\langle x\rangle\rangle, \tau \cong$ $2^{n-1}\langle\langle y\rangle\rangle$ for some $x, y \in \dot{F}$ by (2). If $D(\varphi)=D(\tau)$ then, by (3),

$$
2\langle\langle x\rangle\rangle \cong\langle x, 1, x, 1\rangle \cong\langle x y, y, x, 1\rangle \cong 2\langle x\rangle\langle\langle y\rangle\rangle \cong 2\langle\langle y\rangle\rangle .
$$

Since $n \geqq 2$ this implies $2^{n-1}\langle\langle x\rangle) 2^{n-1}\langle\langle y\rangle\rangle$, i.e. $\varphi \cong \tau$. Q.E.D.

COROLlary. Suppose that $I^{2} F(\sqrt{ }-1)=0$. Then

(1) A form $r\langle\langle x\rangle\rangle, r \geqq 1$ an integer, is anisotropic over $F$ iff $2 r\langle\langle x\rangle\rangle$ is anisotropic or $r=1$ and $x \notin-\dot{F}^{2}$.

(2) A form $2 r\langle\langle x\rangle\rangle, r \geqq 1$ an integer, is anisotropic over $F$ iff $x \notin D(2\langle-1\rangle)$.

Proof. (2) By Theorem 1(3), $D(\langle\langle x\rangle\rangle)=D(r\langle\langle x\rangle\rangle)=D(2 r\langle\langle x\rangle\rangle)$ for any integer $r \geqq 1$ and any $x \in \dot{F}$. Thus $2 r\langle\langle x\rangle\rangle$ is isotropic iff $-1 \in D(\langle\langle x\rangle\rangle)$ iff $x \in D(2\langle-1\rangle)$.

(1) If $r\langle\langle x\rangle\rangle$ is anisotropic then $x \notin-\dot{F}^{2}$. Since a form $\langle\langle w\rangle\rangle, w \in$ $D(2\langle-1\rangle)-\left(-\dot{F}^{2}\right)$ is anisotropic over $F$, (1) follows from (2). Q.E.D.

THEOREM 2. If $I^{2} F(\sqrt{ }-1)=0$ then for any anisotropic form $q \in I^{n} F$, there exist $r_{i}$-fold Pfister forms $\varphi_{i, r_{i}}$ and $x_{i} \in \dot{F}$ such that

$$
q \cong \stackrel{r}{i=1}_{i=1}^{r}\left\langle x_{i}\right\rangle \varphi_{i, r_{i}}
$$

where $n \leqq r_{1} \leqq \cdots \leqq r_{r}$ and no three successive integers $r_{i}$ are the same.

REMARK. The conclusion of Theorem 2 holds in a somewhat more general situation. For details see (2.3.15) of [2].

Proof. Suppose $\varphi=\langle a\rangle\langle\langle x\rangle\rangle \perp\langle b\rangle\langle\langle y\rangle\rangle \perp\langle c\rangle\langle\langle z\rangle\rangle$ is anisotropic. Then, by Theorem 1(1), there exist $t, u, v \in \dot{F}$ such that $\varphi \cong\langle t\rangle\langle\langle x y z\rangle\rangle \perp 2\langle u\rangle\langle\langle v\rangle\rangle$. Hence

$$
2^{n} \varphi \cong 2^{n}\langle t\rangle\langle\langle x y z\rangle\rangle \perp 2^{n+1}\langle u\rangle\langle\langle v\rangle\rangle \text { for any } n \geqq 0 .
$$

Suppose that $q \in I^{n} F$ is anisotropic. Repeated applications of (*) produce $r_{i}$-fold Pfister forms $\varphi_{i, r_{i}}$ and $x_{i} \in \dot{F}$ such that $q \cong \perp_{i=1}^{r}\left\langle x_{i}\right\rangle \varphi_{i, r_{i}}$ where $1 \leqq r_{1} \leqq \cdots \leqq r_{r}$ and no three successive $r_{i}$ are the same. Without loss of generality, we may assume that the $\varphi_{i, r_{i}}$ have also been chosen to satisfy the property that $r_{1}$ is maximal. If $n=1$, we are done, so we 
may assume that $n \geqq 2$. Suppose that $r_{1}<n$. We derive a contradiction. If $r_{1}<r_{2}$ then $\varphi_{1, r_{1}} \in I^{r_{1}+1} F$. The Hauptsatz implies that $q$ is isotropic, a contradiction. Thus $r_{1}=r_{2}$ and $\varphi_{1, r_{1}} \equiv \varphi_{2, r_{2}}\left(\bmod I^{r_{1}+1} F\right)$. Applying the Hauptsatz to the anisotropic part of the form $\varphi_{1, r_{1}} \perp\langle-1\rangle \varphi_{2, r_{2}}$ shows that $\varphi_{1, r_{1}} \cong \varphi_{2, r_{2}}$. Consequently,

$$
q \cong\left\langle x_{1}\right\rangle\left\langle\left\langle x_{1} x_{2}\right\rangle\right\rangle \varphi_{1, r_{1}} \perp \sum_{i=3}^{r}\left\langle x_{i}\right\rangle \varphi_{i, r_{i}} .
$$

Repeated use of Theorem 1(2) and (*) on this form produces $s_{i}$-fold Pfister forms $\psi_{i, s_{i}}$ and $y_{i} \in \dot{F}$ such that $q \cong \perp_{i=1}^{s}\left\langle y_{i}\right\rangle \psi_{i, s_{i}}$ where $r_{1}<$ $s_{1} \leqq \cdots \leqq s_{s}$ and no three successive $s_{i}$ are equal. This contradicts the maximality of $r_{1}$, and proves the theorem. Q.E.D.

Proposition. Suppose that $I^{2} F(\sqrt{ }-1)=0$ and $q \in I^{n} F, n \geqq 2$, is anisotropic. Then $q$ is rund iff there exist a positive integer $r$ and an $x \in \dot{F}-$ $D(2\langle-1\rangle)$ such that $q \cong 2^{n-1} r\langle\langle x\rangle\rangle$. In particular, if $\tau$ is an arbitrary form of dimension $2^{n}$ over $F$, then $\tau$ is an $n$-fold Pfister form iff det $\tau=1$ and $\tau$ is rund.

Proof. By the corollary to Theorem $1,2^{n-1} r\langle\langle x\rangle\rangle, x \notin D(2\langle-1\rangle)$, is anisotropic. Furthermore, it is rund by Theorem 1(3). Hence the form $2^{n-1} r\langle\langle x\rangle\rangle$ has the desired properties. Conversely, suppose that $q \in I^{n} F$ is anisotropic and rund. We may write $q \cong \perp_{i=1}^{r}\left\langle x_{i}\right\rangle \varphi_{i, r_{i}}$ where $x_{i} \in \dot{F}$, $\varphi_{i, r_{i}}$ are $r_{i}$-fold Pfister forms, and the $r_{i}$ satisfy the conclusion of Theorem 2. We claim that we can, in fact, choose such Pfister forms $\varphi_{i, r_{i}}$ such that $2 \leqq n \leqq r_{1}<r_{2}<\cdots<r_{r}$. Suppose not. We may assume that the $\varphi_{i, r_{i}}$ have been chosen. such that $r_{j}$ is maximal if $j$ is the first integer satisfying $r_{j}=r_{j+1}$. We derive a contradiction. Let $x \in D\left(\varphi_{j, r_{j}}\right)$. Then $\langle\langle-x\rangle\rangle q=0$ since $q$ is rund. The Hauptsatz implies that $\langle\langle-x\rangle\rangle \varphi_{j+1, r_{j+1}}=0$. By symmetry, it follows that $D\left(\varphi_{j, r_{j}}\right)=D\left(\varphi_{j+1, r_{j+1}}\right)$. Thus $\varphi_{j, r_{j}} \cong \varphi_{j+1, r_{j+1}}$ by Theorem 1(4). Since $\left\langle x_{j}\right\rangle \varphi_{j, r_{j}} \perp\left\langle x_{j+1}\right\rangle \varphi_{j+1, r_{j+1}} \cong\left\langle x_{j}\right\rangle\left\langle\left\langle x_{j} x_{j+1}\right\rangle\right\rangle \varphi_{j, r_{j}}$, the form $q_{1}=\perp_{i=j}^{r}\left\langle x_{i}\right\rangle \varphi_{i, r_{i}} \in I^{r j+1} F$. Applying Theorem 2 to the form $q_{1}$ contradicts the maximality of $r_{j}$ and establishes the claim. Thus $q \cong$ $\perp_{i=1}^{r}\left\langle x_{i}\right\rangle \varphi_{i, r_{i}}, 2 \leqq n \leqq r_{1}<\cdots<r_{r}$. If $x \in D\left(\varphi_{i, r_{i}}\right)$ then $\langle\langle-x\rangle\rangle q=0$. The Hauptsatz implies that $\langle\langle-x\rangle\rangle \varphi_{j, r_{j}}=0,1 \leqq j \leqq r$. Consequently, $D\left(\varphi_{i, r_{i}}\right)=$ $D\left(\varphi_{j, r_{j}}\right), 1 \leqq i \leqq j \leqq r$. Theorem 1(2)-(4) produces an $x \in \dot{F}$ such that $\varphi_{i, r_{i}} \cong 2^{r_{i}-1}\langle\langle x\rangle\rangle, 1 \leqq i \leqq r$. A further application of the Hauptsatz yields $x_{i} \in G\left(2^{r_{i}-1}\langle\langle x\rangle\rangle\right)$. Thus $q \cong 2^{r_{1}-1} s\langle\langle x\rangle\rangle$ for some integer $s \geqq 1$ and establishes the first statement. If $\tau$ is Pfister it is rund, so we need only prove the converse. If det $\tau=1$ then $\tau \in I^{2} F$. Hence if $\tau$ is anisotropic and rund, $\tau \cong$ $2 r\langle\langle x\rangle\rangle$ for some $x \in \dot{F}$. Comparing dimensions yields $\tau \cong 2^{n-1}\langle\langle x\rangle\rangle$. If $\tau$ is hyperbolic, $\tau \cong 2^{n-1}\langle\langle-1\rangle\rangle$, and the proof of the proposition is complete. Q.E.D. 
REMARK. If $I^{m} F$ is torsion-free and all anisotropic $q \in I^{m} F$ satisfy the conclusion of Theorem 2, then one can classify rund forms over $F$ lying in $I^{m} F$ by the same methods. In particular, this applies to a field $F$ if $F$ is algebraic over $\boldsymbol{Q}$ and $m=3$. In fact, in this case, we can also classify rund forms in $I^{2} F$ by similar methods.

We can now state and prove our classification of even dimensional anisotropic rund forms over a field $F$ if $I^{2} F(\sqrt{ }-1)=0$.

THEOREM 3. Suppose that $I^{2} F(\sqrt{ }-1)=0$ and $q$ is an anisotropic form over $F$. Then

(1) If $\operatorname{dim} q \equiv 2(\bmod 4)$ then $q$ is rund iff $q \cong(2 r+1)\langle\langle x\rangle\rangle$ for some integer $r \geqq 0$, where $x \in \dot{F}-D(2\langle-1\rangle)$ if $r \geqq 1$ and $x \in \dot{F}-\left(-\dot{F}^{2}\right)$ if $r=0$.

(2) If $\operatorname{dim} q \equiv 0(\bmod 4)$ then $q$ is rund iff $q \cong\langle\langle x w\rangle\rangle \perp(2 r+1)\langle\langle x\rangle\rangle$ for some integer $r \geqq 0, w \in D(2\langle 1\rangle)$ and $x \in \dot{F}-D(2\langle-1\rangle)$.

Proof. (1) The forms $(2 r+1)\langle\langle x\rangle\rangle$ in (1) are anisotropic by the corollary to Theorem 1 and rund by Theorem 1(3). Conversely, suppose $q$ is rund and $\operatorname{dim} q \equiv 2(\bmod 4)$. By Theorem $1(1), q \cong\langle y\rangle\langle\langle d\rangle\rangle \perp 2 q_{1}$ for some $y \in \dot{F}$ where $d=\operatorname{det} q$. Our hypotheses on $q$ imply that $q \cong\langle\langle d\rangle\rangle \perp 2\langle y\rangle q_{1}$ and $2\langle y\rangle q_{1} \in I^{2} F$. If $q_{1}$ is the zero form, we are done by the corollary to Theorem 1 , so we may assume that $q_{1}$ is not the zero form. We claim that the anisotropic form $2\langle y\rangle q_{1}$ is rund. If $z \in D(q)=G(q)$, the Hauptsatz yields $z \in G(\langle\langle d\rangle\rangle) \cap G\left(2\langle y\rangle q_{1}\right)$. Hence $D\left(2\langle y\rangle q_{1}\right) \subset D(q)=G(q)=G\left(2\langle y\rangle q_{1}\right)$. It follows easily that $1 \in D\left(2\langle y\rangle q_{1}\right)$ and thus $G\left(2\langle y\rangle q_{1}\right) \subset D\left(2\langle y\rangle q_{1}\right)$. Therefore $2\langle y\rangle q_{1}$ is rund as claimed. By the proposition, $2\langle y\rangle q_{1} \cong 2 r\langle\langle x\rangle\rangle$ for some $x \in \dot{F}-D(2\langle-1\rangle)$ and some integer $r \geqq 1$. The Hauptsatz yields

$$
D(2 r\langle\langle x\rangle\rangle)=D(\langle\langle d\rangle\rangle),
$$

and Theorem 1(3), (4) yields $2\langle\langle x\rangle) 2\langle\langle d\rangle\rangle$. Thus $q \cong(2 r+1)\langle\langle d\rangle\rangle$. If $d \in D(2\langle-1\rangle)$ then $r=0$ by the corollary to Theorem 1 , the case previously done. This establishes (1).

(2) Since $D(r\langle\langle x\rangle\rangle)=D(\langle\langle x\rangle\rangle)$ for any $x \in \dot{F}$ by Theorem 1(3), we have $G(\langle\langle x\rangle\rangle)=D(\langle\langle x\rangle\rangle)=D(\langle\langle w x\rangle\rangle)=G(\langle\langle w x\rangle\rangle)$. Thus the forms $\langle\langle x w\rangle\rangle \perp$ $(2 r+1)\langle\langle x\rangle\rangle$ are rund. They are anisotropic by the corollary to Theorem 1 . Conversely, suppose that $\operatorname{dim} q \equiv 0(\bmod 4)$ and $q$ is rund. Using Theorem $1(1)$ and the rund property yields an isometry $q \cong\langle\langle d\rangle\rangle \perp\langle x, x\rangle \perp q_{0}$ for some $x \in \dot{F}$ and $q_{0} \in I^{2} F$, where $d=\operatorname{det} q$. Since $\langle\langle-d\rangle\rangle q=0$, we have $\langle x\rangle\langle\langle 1,-d\rangle\rangle=0$ by the Hauptsatz. This shows that $d \in D(2\langle 1\rangle)$.

Case 1. $2 q$ is anisotropic. Since $d \in D(2\langle 1\rangle)$,

$$
\begin{aligned}
2 q & \cong\langle\langle d, 1\rangle\rangle \perp\langle x\rangle\langle\langle 1,1\rangle\rangle \perp 2 q_{0} \\
& \cong\langle\langle 1,1\rangle\rangle \perp\langle x\rangle\langle\langle 1,1\rangle\rangle \perp 2 q_{0} \\
& \cong\langle\langle x, 1,1\rangle\rangle \perp 2 q_{0} \in I^{3} F=4 I F .
\end{aligned}
$$


But $G(2 q) \subset D(2 q)=D(q)=G(q) \subset G(2 q)$. Consequently, the anisotropic form $2 q$ is also rund. By the proposition, $2 q \cong 4 r\langle\langle x\rangle\rangle$ for some integer $r \geqq 1$ and $x \in \dot{F}-D(2\langle-1\rangle)$. By (4.11) of [3], any anisotropic torsion element in $W(F)$ must have dimension $\leqq 2$. Therefore, by (2.2) of [3]

$$
q-2 r\langle\langle x\rangle\rangle=\langle y\rangle\langle\langle-w\rangle\rangle=\langle\langle-w\rangle\rangle
$$

in $W(F)$ for some $w \in D(2\langle 1\rangle)$. If $w \in \dot{F}^{2}$ then $q \cong 2 r\langle\langle x\rangle\rangle$ and the result follows, so we may further assume that $w \notin \dot{F}^{2}$. Therefore,

$$
\begin{aligned}
q & =2(r-1)\langle\langle x\rangle\rangle+\langle-w, 1,1,1, x, x\rangle \\
& =2(r-1)\langle\langle x\rangle\rangle+\langle w, 1, x, x\rangle
\end{aligned}
$$

in $W(F)$. Comparing dimensions yields the isometry

as desired.

$$
\begin{aligned}
q & \cong 2(r-1)\langle\langle x\rangle\rangle \perp\langle w, 1, x, x\rangle \cong\langle x\rangle q \\
& \cong 2(r-1)\langle\langle x\rangle\rangle \perp\langle x\rangle\langle w, 1, x, x\rangle \\
& \cong(2 r-1)\langle\langle x\rangle\rangle \perp\langle\langle w x\rangle\rangle
\end{aligned}
$$

Case 2. $2 q$ is isotropic. By (2.2) of [3], there exists a $w \in D(2\langle 1\rangle)$ such that $q \cong\langle\langle-w\rangle\rangle \perp q_{1}$. Applying Theorem 1(1) to the form $q_{1}$ allows us to write $q_{1}=\langle y\rangle\langle\langle-w d\rangle\rangle \perp 2 q_{2}$. But $w d \in D(2\langle 1\rangle)$ implies that $\langle\langle-w d\rangle\rangle$ is a torsion element in $W(F)$. Since $I^{2} F$ is torsion-free by Theorem $1(2)$, it follows that $\langle\langle-y,-w d\rangle\rangle=0$ in $W(F)$, i.e. $\langle y\rangle\langle\langle-w d\rangle) \cong\langle\langle-w d\rangle\rangle$. Hence $\langle\langle-w\rangle\rangle \perp\langle y\rangle\langle\langle-w d\rangle\rangle \cong\langle-1\rangle\langle\langle-w\rangle\rangle \perp\langle\langle-w d\rangle\rangle$ is isotropic. Consequently, $q$ is isotropic, a contradiction. This establishes (2). Q.E.D.

\section{REFERENCES}

1. J. K. Arason and A. Pfister, Beweis des Krullschen Durchschnittsatzes für den Wittring, Invent. Math. 12 (1972), 173-176. MR 45 \#3320.

2. R. Elman, Pfister forms and $K$-theory of fields, Thesis, University of California, Berkeley, Calif., 1972.

3. R. Elman and T. Y. Lam, Quadratic forms and the u-invariant. I, Math. Z. 131 (1973), 283-304.

4. J. Hsia and R. P. Johnson, Round and group quadratic forms over global fields, J. Number Theory (to appear).

5. - - Round and Pfister forms over $\boldsymbol{R}(t)$ (preprint).

6. W. Scharlau, Quadratic forms, Queen's Papers in Pure and Appl. Math., no. 22, Queen's University, Kingston, Ont., 1969. MR 42 \#4574.

Department of Mathematics, Rice University, Houston, TeXas 77001

Current address: Department of Mathematics, University of California, Los Angeles, California 90024 